\title{
Three-Dimensional Hysteresis Modeling of Robotic Artificial Muscles with Application to Shape Memory Alloy Actuators
}

\author{
Jun Zhang and Michael C. Yip \\ Department of Electrical and Computer Engineering \\ University of California San Diego, La Jolla, CA, USA, \{j5zhang, yip $\} @$ ucsd.edu
}

\begin{abstract}
Being inherently compliant, the robotic artificial muscles are increasingly popular in applications such as safe human-robot interaction, legged robotics, prostheses and orthoses, and soft robotics. Their full utilization is often challenged by the coupled hysteresis among input, strain, and tension force. Although conventional two-dimensional hysteresis models are available, no prior studies on three-dimensional hysteresis models with coupled inputs have been reported for robotic artificial muscles. This paper presents a new approach to capturing the threedimensional hysteresis of robotic artificial muscles by embedding a two-stage Preisach model. The proposed method is applied to shape memory alloy (SMA) actuators. Since direct temperature measurement of the SMA actuator is not available, the concept of temperature surrogate, representing the constant voltage value in Joule heating that would result in a given temperature at the steady-state, is adopted. The proposed approach is utilized to capture the hysteresis among temperature surrogate, contraction length, and force of an SMA actuator. Model verification is further conducted. For comparison purposes, two modeling approaches, namely, the Summed Preisach and the Linear Preisach, are also realized. Experimental results demonstrate that the proposed scheme can effectively characterize and estimate the three-dimensional hysteresis in SMA actuators. This study can be applied towards other robotic artificial muscles such as McKibben actuators and Super-coiled Polymer actuators.
\end{abstract}

\section{INTRODUCTION}

Robotic artificial muscles are actuators that have similar working mechanisms as biological muscles - they can contract in their cross-sectional directions when activated [8, 26]. Compared to conventional actuators such as electric motors and hydraulic actuators, robotic artificial muscles have demonstrated high power-to-weight ratio, high force-to-weight ratio, inherent compliance, and good dynamic range in a muscle-like form factor [8, 26, 27]. While electric motors and hydraulic actuators can produce large forces and achieve accurate positioning, they often introduce substantial mass, inertia and friction, may require large volumes, and rely on linkages or substantial gear reductions to drive joints. Therefore, they generally do not exhibit muscle-like properties. The robotic artificial muscles, such as shape memory alloy (SMA) actuators, McKibben actuators, and Super-coiled Polymer (SCP) actuators (Fig. 1), are increasingly popular in novel robotic applications such as safe human-robot interaction, legged robotics, prostheses and orthoses, and soft robotics [8, 14, 22, 32, 36]. However, it is often a challenging task to accurately model and control these actuators due to the three-dimensional and coupled hysteresis nonlinearities - any of the two variables among input, strain, and tension force are often mutually correlated. No prior studies on three-dimensional hysteresis models with coupled variables have been reported for robotic artificial muscles.

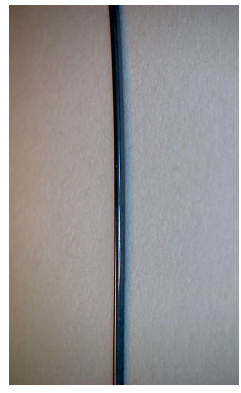

(a)

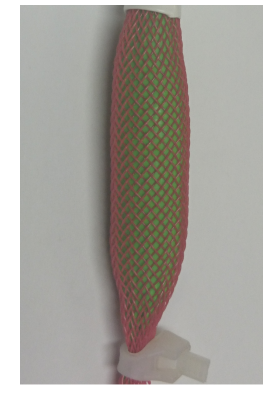

(b)

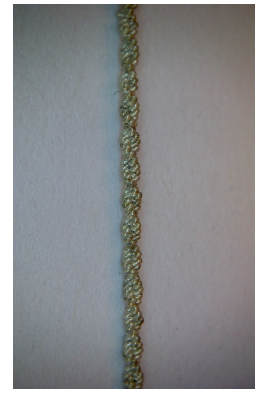

(c)
Fig. 1. (a). A Flexinol shape memory alloy (SMA) actuator (Dynalloy, Inc). Diameter: $0.38 \mathrm{~mm}$. (b). A McKibben actuator. Diameter: $8 \mathrm{~mm}$. (c). A Super-coiled Polymer (SCP) actuator. Diameter: $0.62 \mathrm{~mm}$.

SMAs are a group of metallic materials that can recover the strain under certain stimulus such as thermomechanical variations [10]. They have been adopted in various areas, such as automotive, aerospace, micro-electromechanical systems, and robotics [9, 21, 23]. However, the three-dimensional and coupled hysteresis among temperature, strain, and force in SMA actuators has not been accurately captured [10]. Although physics-based models have been proposed, the analysis is often constrained to particular types of SMAs and and could not faithfully capture the hysteresis [9]. The derivation of these models is based on complicated molecular-level physics [4]. Phenomenological models that are derived directly based on input and output data are proposed, however, the existing models can only capture the hysteresis between two domains and were thus limited in many applications where strain and tension force varied simultaneously. For example, an adaptive neuro-fuzzy inference system model was realized for an SMA actuator at various frequencies, but only the voltage - strain hysteresis was captured [12]. Similarly, a generalized PrandtlIshlinskii model was adopted for position control, only the temperature - deflection hysteresis was compensated [33]. In practical applications, such as SMA-actuated robot hand for grasping object with different temperature and weight, the 
strain and tension force of the SMA actuators would vary under varying loads and inputs, resulting in complicated coupling relationships. The existing methods could not faithfully capture or compensate for the three-dimensional and coupled hysteresis in SMA actuators.

Modeling of hysteresis has been an active research area, and the existing studies have predominantly focused on twodimensional hysteresis scenarios. Since physics-based models are derived based on material properties [4], their utilization is more limited comparing to phenomenological models (e.g., Preisach model, Krasnoselskii-Pokrovskii model, Prandtl-Ishlinskii model, Maxwell-Slip model, Duhem model, and Bouc-Wen model), that are directly obtained by input and output data [1, 15, 18, 24, 28]. Among the phenomenological models, Preisach model has proven to be effective for various hysteresis behaviors [18, 25, 34, 35]. The Preisach model can be expressed as a weighted superposition of delayed relays. Practical model implementation involves discretization of Preisach weight function to obtain a finite number of parameters [19, 25]. The identification of the Preisach model could often be reformulated as a linear least-squares problem and solved efficiently [25, 34]. The Preisach model is adopted in this work considering its great advantages, however, the hysteresis in robotic artificial muscles are three-dimensional and coupled, new methods need to be developed.

Comparing to the modeling of two-dimensional hysteresis, describing higher-dimensional hysteresis is significantly more challenging. Few strategies exist dealing with multi-variable hysteresis. Finocchio et al. [6] proposed a vector hysteresis model based on the Preisach model to capture the isotropic hysteresis behavior of the magnetization process, the magnetization output was computed in two steps to obtain the amplitude and phase independently. Similarly, Krejci and Sprekels [13] presented a multi-dimensional Prandtl-Ishlinskii model, but the model assumed that each input variable independently influenced the output. The existing multi-dimensional hysteresis models cannot be directly adopted to describe the hysteresis in robotic artificial muscles, where the hysteresis among input, strain, and force are mutually coupled.

This paper presents the first study to capturing the threedimensional and coupled hysteresis of robotic artificial muscles by embedding a two-stage Preisach model. The proposed method is applied to SMA actuators. Since direct temperature measurement of SMA actuators is not available, the concept of temperature surrogate, representing the constant voltage value in Joule heating that would result in a given temperature at the steady-state, is adopted. The proposed approach is utilized to capture and estimate the hysteresis among temperature surrogate, contraction length, and force of an SMA actuator. For comparison purposes, two modeling alternatives, namely, the Summed Preisach and the Linear Preisach, are also realized. Experimental results demonstrate that the proposed scheme can effectively characterize and estimate the three-dimensional hysteresis of the SMA actuator simultaneously. For example, The root-mean-square (RMS) modeling percentage errors for Length - Force hysteresis are $4.9 \%, 6.3 \%$, and $11.6 \%$, under the proposed model, Summed Preisach, and Linear Preisach models, respectively. This work can be applied towards other artificial muscles such as McKibben actuators and SCP actuators.

\section{Characterization AND Discussion}

\section{A. Characterization}

SMAs are a group of metallic materials with the capability of returning to the previous shape under temperature or stress stimulus. When the shape memory effect occurs, the crystalline structure of the material changes between Martensite phase and Austenite phase [10]. While SMA actuators have been adopted in various applications, the accurate modeling is challenging due to their coupled hysteresis among temperature, strain, and force. More details can be found in [4, 10].

To fully characterize the hysteresis in SMA actuators, three static relationships need to be jointly considered, namely, the Force - Voltage hysteresis under constant length, the Length - Voltage hysteresis under constant force, and the Contraction Length - Force hysteresis under constant voltage. Denote the contraction length $L$, voltage $V$, and force $F$.

1) F-V Hysteresis: The relationship between the steadystate force and voltage of the actuator was measured when the actuator length was close to resting length. In order to prevent that the SMA actuator being slack under low voltage, a pretension of $0.3 \mathrm{~N}$ was used. The force was measured by a load cell. Since the time constant of the thermal dynamics was verified to be approximately $5 \mathrm{~s}$, each voltage step was held for $30 \mathrm{~s}$, ensuring that the steady-state temperature and force have been reached. Note that the dynamics of the actuator has been explored [9, 23]. Fig. 2 (left) shows the corresponding hysteresis measurements. The force range is $[0.3,11.2] \mathrm{N}$, and the voltage range is $[0,3] \mathrm{V}$.

2) L-V Hysteresis: Fig.2 (middle) shows a series of major hysteresis curves between quasi-static contraction length and voltage under different loading forces ranging from $0.98 \mathrm{~N}$ to $8.82 \mathrm{~N}$. Loading forces were generated by attaching weights to the actuator, and the contraction length was measured by a position sensor. The contraction length was defined as the change of actuator length with respect to its resting length. The contraction length increases when the actuator is elongated, and decreases during contraction. Since the actuator length changes with different loading forces, the initial value of each curve is different. It is noticed that the hysteresis curves exhibit different shapes, further challenging the accurate modeling with efficient methods.

3) $L-F$ Hysteresis: The relationship between steady-state contraction length and force of the actuator was measured under different constant voltages. The contraction length was controlled by programming a servo motor that was attached to the actuator. Fig. 2 (right) shows a series of major hysteresis curves. The initial forces among different hysteresis curves are close to zero, and the corresponding lengths of hysteresis curves are different. This is due to the fact that, at higher voltages, the actuator has a shorter length under contraction. The final length of the actuator is determined such that the 

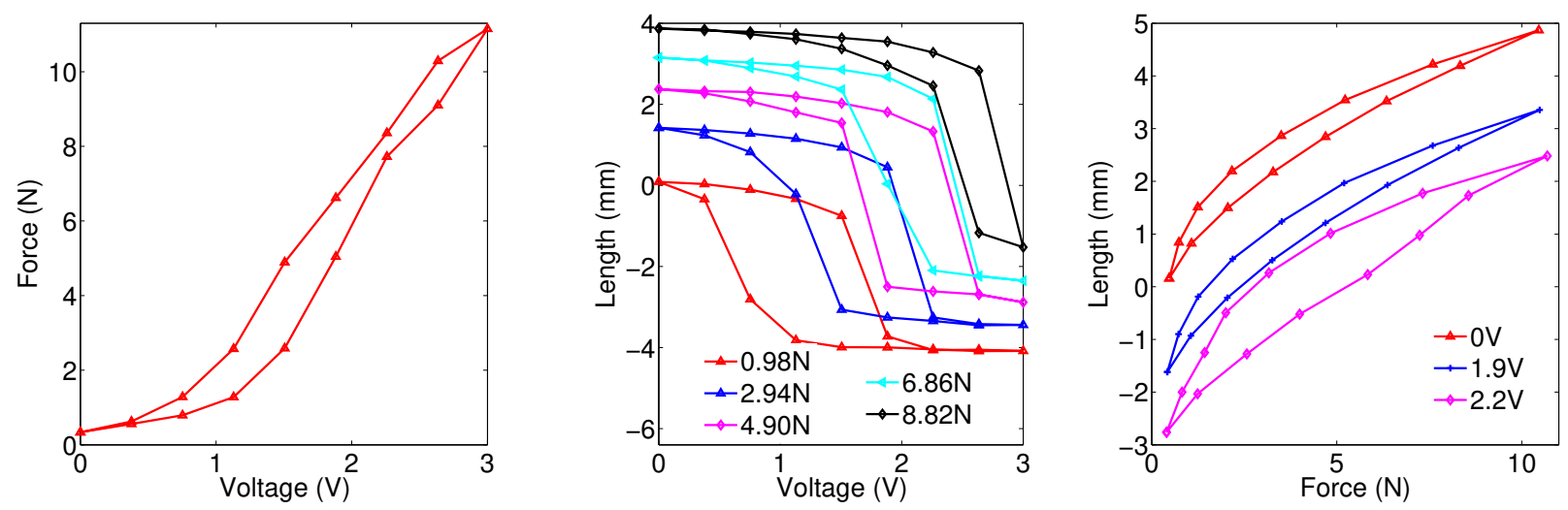

Fig. 2. The major hysteresis curves of the $F-V$ hysteresis (left), $L-V$ hysteresis (middle), and $L-F$ hysteresis (right) of an SMA wire actuator.

largest forces under different experiments are approximately the same.

\section{B. Steady-state Voltage as Temperature Surrogate}

The phase transition of SMA is induced by temperature, for example, the phase transition of the SMA actuator in this work occurs within $[70,90]^{\circ} \mathrm{C}$. Since the adopted SMA wire is too thin $(0.20 \mathrm{~mm}$ in diameter $)$ for conventional thermocouples or laser thermometers to work properly, direct temperature measurement of the SMA actuator is a challenging task. In order to obtain the temperature information, in this work, the quasi-static voltage $V$ is adopted as the temperature surrogate [35]. The goal of applying quasi-static voltage inputs is to achieve the steady-state temperature values, and further obtain the relationship between contraction length/force and temperature. The applied quasi-static voltage input value, $V$, becomes a surrogate for the steady-state temperature $T$, since $V$ can be formulated as a single-valued and monotonically increasing function of $T$, namely, $V=q(T)$.

Although the explicit expression of the temperature surrogate, $q(T)$, is not required for model implementation, an illustrative example is provided here based on a simple thermal model of Joule heating [16]. The thermo-electric model of the SMA actuator can be expressed as

$$
\frac{d T(t)}{d t}=-d_{1}\left(T(t)-T_{0}\right)+d_{2} V^{2}(t)
$$

where $d_{1}$ and $d_{2}$ are positive constants relevant to the density, volume, heat transfer coefficient, resistance, and surface area of the SMA actuator, and $T_{0}$ is the ambient temperature. Note that the resistance of the actuator might also undergo mild changes during phase transition, the change is less than $15 \%$ [29] and is considered to be constant in this work.

Under a constant (quasi-static) voltage $V$, the steady-state temperature $T$ can be computed as $\frac{d_{2}}{d_{1}} V^{2}(t)+T_{0} . V$ can be expressed in terms of $T$ :

$$
V=\sqrt{\frac{d_{1}}{d_{2}}\left(T-T_{0}\right)}=q(T)
$$

$q(T)$ in Eq. (2) is indeed single-valued and strictly increasing, and thus is a legitimate surrogate for $T$.

\section{Hysteresis MOdeL}

From Fig. 2, it is shown that the relationship among temperature surrogate, contraction length, and force of the SMA actuator is hysteretic and coupled together. The hysteresis curves exhibit different shapes under different conditions. Individual hysteresis model could be separately identified and then combined to obtain the overall model. However, then the model will be overly complicated.

In this section, an efficient model is proposed for capturing the three-dimensional and coupled hysteresis in SMA actuators. the proposed model is derived by embedding a two-stage Preisach model: the proposed approach first characterizes the $F-q(T)$ hysteresis as $F=H_{1}[q(T)]$ when the actuator is close to its resting length, and then embeds the relationship into the overall model as $L=H_{2}\left[F-H_{1}[q(T)]\right]$. Two alternative methods, namely, the Summed Preisach and the Linear Preisach, are also briefly described. More details on Preisach model can be found in [18, 19, 25, 34].

\section{A. Force - Temperature Surrogate Hysteresis}

A Preisach model with non-negative weight function $\omega$ is adopted to model the $F-q(T)$ hysteresis:

$$
\begin{aligned}
F(t) & =H_{1}\left[q(T(\cdot)) ; \zeta_{0}\right](t) \\
& =\int_{\mathscr{P}_{0}} \omega(\beta, \alpha) \gamma_{\beta, \alpha}\left[q(T(\cdot)) ; \zeta_{0}(\beta, \alpha)\right](t) \mathrm{d} \beta \mathrm{d} \alpha+c_{0},
\end{aligned}
$$

where $\mathscr{P}_{0}$ is called the Preisach plane $\mathscr{P}_{0} \triangleq\{(\beta, \alpha)$ : $\left.q\left(T_{\min }\right) \leq \beta \leq \alpha \leq q\left(T_{\max }\right)\right\}$, where $\left[q\left(T_{\min }\right), q\left(T_{\max }\right)\right]$ is the range of the temperature surrogate, which equals to the steadystate voltage range. $\gamma_{\beta, \alpha}$ denotes the hysteron, which is the basic element of the Preisach model, $c_{0}$ is a constant bias. The length of the actuator is maintained close its resting length and a pretension of $0.3 \mathrm{~N}$ is used (see Section II.A), so the initial condition $H_{1}(0)=0.3 \mathrm{~N}$ is held.

The output of the hysteron at time $t$ depends on the history of temperature surrogate up to time $t: q(T(\tau)), 0 \leq \tau \leq t$. $\zeta_{0}(\beta, \alpha) \in\{-1,1\}$ is the initial hysteron output, the hysteron 
at $t$ can be expressed as:

$\gamma_{\beta, \alpha}\left[q(T(\cdot)) ; \zeta_{0}(\beta, \alpha)\right](t)= \begin{cases}+1, & \text { if } q(T(t))>\alpha \\ -1, & \text { if } q(T(t))<\beta \\ \zeta_{0}(\beta, \alpha), & \text { if } \beta \leq q(T(t)) \leq \alpha\end{cases}$

In practical usage, the integral expression of the Preisach model is approximated by discretizing the model parameters (weight function) $\omega$ to a finite number of parameters [19, 25]. The weight function is approximated as a piecewise constant function - the weight $w_{i j}$ is constant within cell $(i, j), i=1,2, \cdots, N_{1} ; j=1,2, \cdots, N_{1}-i+1$, where $N_{1}$ is called the discretization level and $w_{i j}$ is the model parameter. Fig. 3 illustrates the Preisach weight function discretization with $N_{1}=4$, as an example of Preisach model discretization.

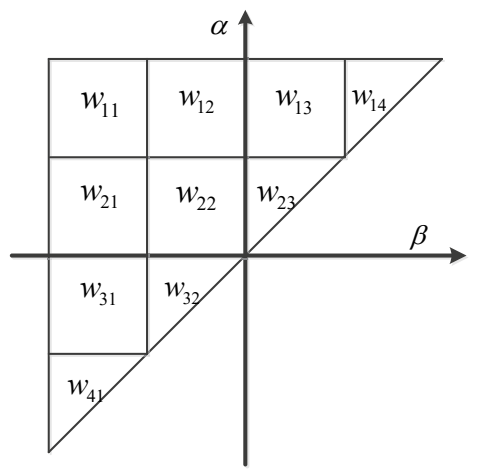

Fig. 3. Illustration of the Preisach weight function discretization.

At time $n$, the output of the discretized Preisach model is expressed as

$$
F(n)=H_{1}[q(T)](n)=\sum_{i=1}^{N_{1}} \sum_{j=1}^{N_{1}+1-i} w_{i j} s_{i j}(n)+c_{0},
$$

where $w_{i j}$ is the weight for the cell $(i, j)$, and $s_{i j}(n)$ is the signed area of the cell $(i, j)$, which is fully determined by the temperature surrogate up to time $n$. Note that $L \approx 0$ and $L$ was assumed not present in the model.

The model parameters consist of the weights $\left\{w_{i j}\right\}$ and the constant bias $c_{0}$. The model identification can be reformulated as a constrained linear least-squares problem and solved efficiently with the Matlab command lsqnonneg [25, 34].

\section{B. Proposed Model}

By embedding the Force - Temperature Surrogate hysteresis ( $F=H_{1}[q(T)]$ ), the following model is proposed to characterize the hysteresis of SMA actuators among temperature surrogate, contraction length, and force:

$$
L(n)=H_{2}\left[F-H_{1}[q(T)]\right](n)=\sum_{i=1}^{N_{2}} \sum_{j=1}^{N_{2}+1-i} \mu_{i j} p_{i j}(n)+c_{1} .
$$

Note that $F(n)=H_{1}[q(T(n))]$ (Eq. 5 held only when $L(n)$ was zero. $H_{2}$ denotes the overall hysteresis model, $N_{2}$ is the model discretization level, $\left\{\mu_{i j}\right\}$ are model parameters, and $c_{1}$ is a constant bias. The negative term of $H_{1}[q(T)]$ is introduced due to the fact that the hysteresis between length and temperature surrogate is monotonically decreasing, as shown in Fig. 2 (middle). Unlike that $\left\{s_{i j}\right\}$ in Eq. (5) are only dependent on the temperature surrogate, $\left\{p_{i j}\right\}$ depend on both temperature surrogate and force. When the loading force is zero, and the actuator temperature equals to room temperature, the actuator will remain its resting length, the initial condition $H_{2}(0)=0$ is held.

It is noted that the identification of the proposed model (Eq. (6) ) can also be realized by the linear least-squares method, and a brief explanation is provided. For ease of presentation, the model parameters are combined: $D=$ $\left(\begin{array}{lllll}d_{1} & d_{2} & \cdots & d_{N_{2}\left(N_{2}+1\right) / 2} & c_{1}\end{array}\right)^{\top}$, where $d_{k}=\mu_{i j}, k=(i-$ 1) $\left(2 N_{2}-i+2\right) / 2+j-1$. The input sequence is denoted as $F[n]-H_{1}[q(T[n])], n=1,2, \cdots, N$, and the corresponding $p_{i j}[n]$ can be calculated by tracking the evolution of the input history. By stacking $p_{i j}[n]$ into a row of a matrix: $P(n, k)=p_{i j}(n)$, and $P\left(n, N_{2}\left(N_{2}+1\right) / 2+1\right)=1$. The output of the model $\tilde{L}=\left(\begin{array}{llll}\tilde{L}(1) & \tilde{L}(2) & \cdots & \tilde{L}(N)\end{array}\right)^{\top}$ can be expressed as

$$
\tilde{L}=P D \text {. }
$$

Denote the experimental contraction length measurements under input sequence $F[n]-H_{1}[q(T[n])], n=1,2, \cdots, N$, are expressed as $Y=\left(\begin{array}{llll}y(1) & y(2) & \cdots & y(N)\end{array}\right)^{\top}$. The parameter $D$ can be calculated such that $\|P D-Y\|_{2}$ is minimized under the constraint that all of the weights are non-negative [25. 36]. Note that the identification of this model assumes the knowledge of $H_{1}$. In practice, $H_{1}$ is firstly identified based on $F-q(T)$ hysteresis, $H_{2}$ is then identified jointly by $L-q(T)$ and $L-F$ hysteresis measurements.

\section{Comparison Methods}

Two alternative methods, namely the Summed Preisach and the Linear Preisach, are briefly discussed.

1) Summed Preisach: The Summed Preisach model considers that the contraction length of the SMA actuator can be expressed as the weighted summation of two independent hysteresis models, $\mathrm{H}_{3}$ and $\mathrm{H}_{4}$, regarding temperature surrogate and force, respectively,

$$
L=H_{3}[F]-H_{4}[q(T)] .
$$

Similarly, the negative term of $H_{4}[q(T)]$ is introduced considering that the hysteresis between contraction length and temperature surrogate is monotonically decreasing (Fig. 2). In order the identify the model parameters, the hysteresis measurements between $L-q(T)$ and $L-F$ can be jointly utilized.

Note that this approach cannot capture the shape difference of each hysteresis curves, for example, when the force $F$ changes to another constant value $F_{0}$, the $L-q(T)$ hysteresis profile will remain the same except with an added offset 
$\left(H_{3}\left[F_{0}\right]-H_{3}[F]\right)$. Furthermore, the $F-q(T)$ hysteresis measurements are not utilized for model identification, thus the model cannot accurately characterize the coupling between the force and temperature surrogate.

2) Linear Preisach: By simplifying the hysteresis between $F$ and $q(T)$ as a linear relationship, the Linear Preisach model is expressed as

$$
L=H_{5}\left[F-a \cdot q(T)-F_{0}\right],
$$

where $H_{5}$ is a Preisach model, the linear term $a$ and offset $F_{0}$ can be identified with a linear regression method using MATLAB command polyfit, and then the hysteresis model $H_{5}$ can be identified based on $L-F$ hysteresis and $L-q(T)$ hysteresis measurements. Since this approach simplifies the $F-q(T)$ hysteresis to be hysteresis-free and linear, significant modeling errors are expected.

\section{EXPERIMENTAL SETUP}

The experimental testbed, as shown in Fig. 4, consists of an SMA actuator (Dynalloy, Inc) with resting length of $105 \mathrm{~mm}$ and diameter of $0.20 \mathrm{~mm}$, a position sensor (SPS-L035-LATS, Honeywell) with $0.04 \mathrm{~mm}$ resolution in contraction length measurements, a load cell (LSP-2, Transducer Techniques) for force measurement, a servo motor (MX-28, Dynamixel) to control the length of the actuator. While SMA actuators with different diameters can be utilized, the one with $0.20 \mathrm{~mm}$ diameter is chosen since they can produce relatively large force with fast response. Similar to [32, 36], a tangential blower fan (QG030-198/12, Ebm-papst) is used to provide an even airflow environment. Fig. 4 a) shows the setup for Contraction Length - Temperature Surrogate hysteresis measurement, and Fig. 4(b) shows the setup for Force - Temperature Surrogate and Contraction Length - Force hysteresis measurements. The SMA actuator has a dark blue color and is highlighted in Fig. 4. The position sensing mechanism is to measure the position of a magnet accurately by an array of magnetoresistive sensors. The magnet and the weights are hung on the actuator to generate loading forces. The weights containing ferrous metals are kept a distance from the magnet to ensure accurate position measurements. The additional mass of the magnet and components hung on the actuator is about $20 \mathrm{~g}$. Quasi-static voltage steps are supplied by a pulse-width-modulated (PWM) circuit. Data acquisition is realized in Labview.

\section{EXPERIMENTAL RESULTS}

In order to examine the proposed method for characterizing and estimating the hysteresis in SMA actuators, model identification and verification experiments are conducted. The performance is measured by the average and RMS of the absolute errors divided by the corresponding output range:

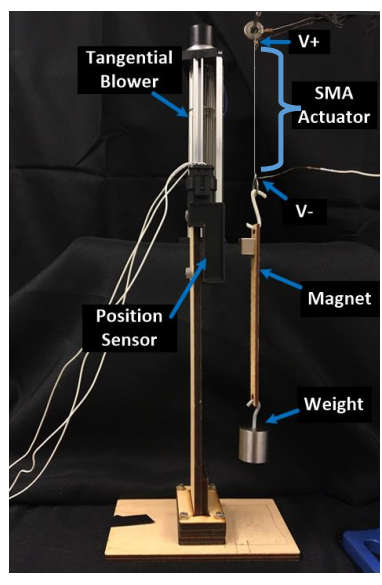

(a)

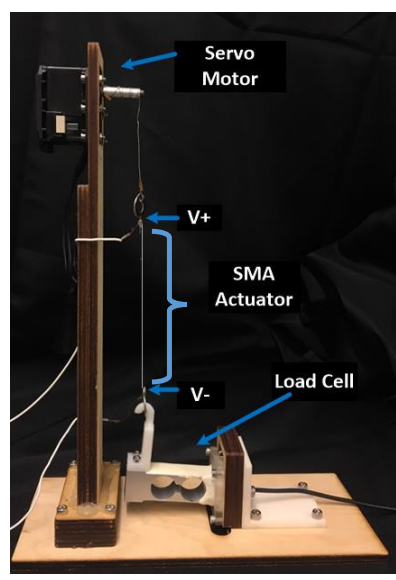

(b)
Fig. 4. The experimental setup for (a) Contraction Length - Temperature Surrogate, and (b) Contraction Length - Force and Force - Temperature Surrogate hysteresis measurements.

$$
\begin{gathered}
E_{\text {average }}=\frac{\sum_{i=1}^{M}\left|e_{i}\right| / M}{R} \times 100 \%, \\
E_{\mathrm{RMS}}=\frac{\sqrt{\sum_{i=1}^{M} e_{i}^{2} / M}}{R} \times 100 \%,
\end{gathered}
$$

where $e_{i}$ denotes the error, $M$ is the number of data-points to be evaluated, and $R$ is the output range. They are denoted as the "average error" and the "RMS error" in this paper. Since quasi-static measurements are taken and the temperature surrogate is obtained based on the steady-state voltage steps, the time information is of less relevance, instead, the term "index" is adopted that refers to the numbering of the quasistatic contraction length, force, and temperature surrogate.

\section{A. Experimental Measurements}

Similar to [19, 25, 35, 36], a damped oscillation-type of voltage input is employed, as shown in Fig. 5 . Each voltage step lasts $30 \mathrm{~s}$ and the steady-state values of the force and the contraction length are recorded. The steady-state voltage values are considered as the temperature surrogate (see Section II.B).

Under resting length condition, a single hysteresis curve between quasi-static force and temperature surrogate is obtained, as shown in Fig. 6. The same voltage input is utilized and the hysteresis between contraction length and temperature surrogate under constant loading forces is shown in Fig. 7 Furthermore, the hysteresis between contraction length and force under constant temperature surrogate values is shown in Fig. 8 It is shown in Fig. 7 and Fig. 8 that hysteresis curves exhibit different profiles under different conditions, and this phenomenon needs to be captured. 


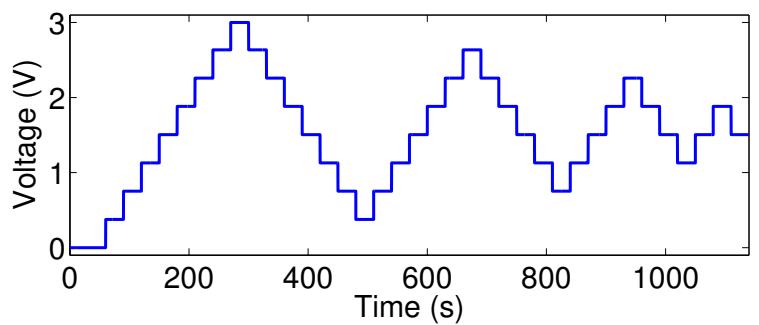

Fig. 5. The voltage input for model identification.
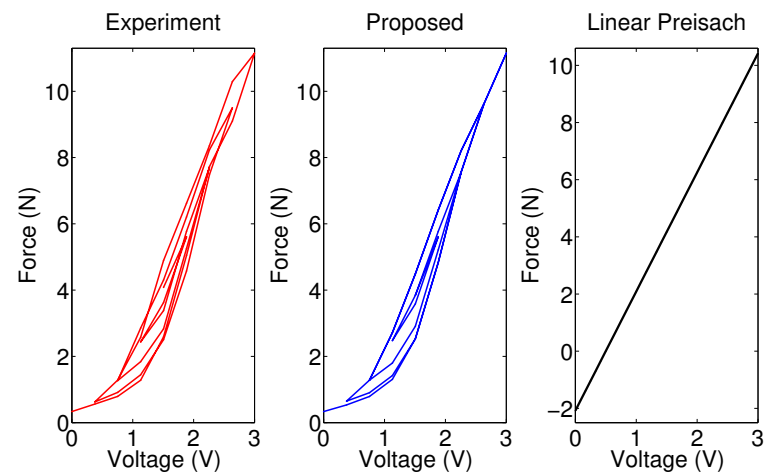

Fig. 6. The experimental hysteresis measurements between steady-state force and temperature surrogate when the actuator is close to resting length (left), and modeling performances of the proposed model (middle) and the Linear Preisach method (right).
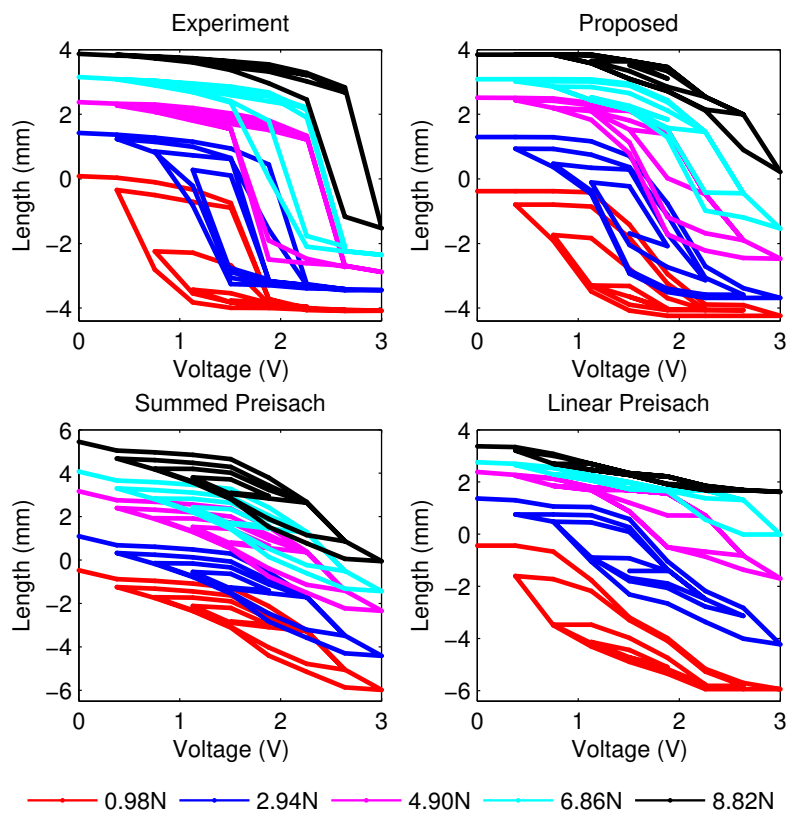

Fig. 7. The experimental hysteresis measurements between steady-state contraction length and temperature surrogate under different loading forces (upper left), and modeling performances of the three modeling approaches.
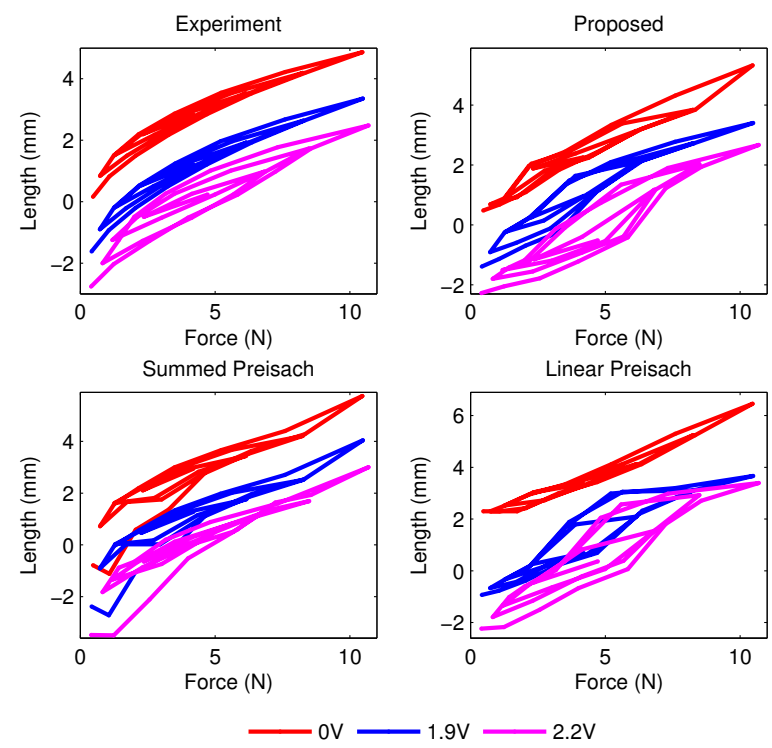

Fig. 8. The experimental hysteresis measurements between steady-state contraction length and force under different temperature surrogate values (upper left), and modeling performances of the three schemes.

\section{B. Model Identification}

1) Proposed Model: In order to identify the proposed model, the discretization levels $\left(N_{1}\right.$ and $\left.N_{2}\right)$ of the Preisach models $H_{1}$ and $H_{2}$ are both set to be 20 since further increasing the discretization levels would increase the model complexity, but do not generate significant improvement in modeling accuracy. $H_{1}$ and $H_{2}$ are identified sequentially.

$H_{1}$ is first identified based on the experimental Force Temperature Surrogate hysteresis (Fig. 6 (left)). Fig. 6 (middle) shows that the proposed model can accurately capture the hysteresis. Fig. 9 (left) shows the identified weights of $H_{1}$. Since the hysteresis between force and temperature surrogate is relatively small, the identified weights of the Preisach model $H_{1}$ are primarily located close to the $\beta=\alpha$ line. While this indeed means that a linear model can be adopted [19, 34], it will be shown later that the adoption of a hysteresis model still produces better modeling performance than using a linearized approach. The average error and RMS error are $1.2 \%$ and $1.9 \%$, respectively.

$\mathrm{H}_{2}$ is then identified based on Contraction Length - Temperature Surrogate hysteresis (Fig. 7) and Contraction Length - Force hysteresis (Fig. 8). The model weights are shown in Fig. 9 (right). Since the hysteresis between contraction length and temperature surrogate is evident, a significant portion of the major weights of the Preisach model $\mathrm{H}_{2}$ are located outside of the $\beta=\alpha$ line [19]. The weights of $H_{2}$ tend to be small at high $\alpha$ and $\beta$ values, contributing to the saturating feature of the hysteresis. The modeling performance is shown in Fig. 7) Fig. 8, and Fig. 10 The average and RMS errors for the Contraction Length - Temperature Surrogate hysteresis are $4.8 \%$ and $7.2 \%$, respectively. The average and RMS errors for the Contraction Length - Force hysteresis are $4.3 \%$ and 
$4.9 \%$, respectively. Since 37 different voltage steps are used in each loading condition (Fig. 5) and 5 loading conditions are tested, a total of 185 data points are adopted for the Contraction Length - Temperature Surrogate modeling error analysis in Fig. 10 a). Similarly, 138 data points are adopted for the Contraction Length - Force modeling error analysis in Fig. 10, b). The model can effectively capture the hysteresis of the SMA actuator.
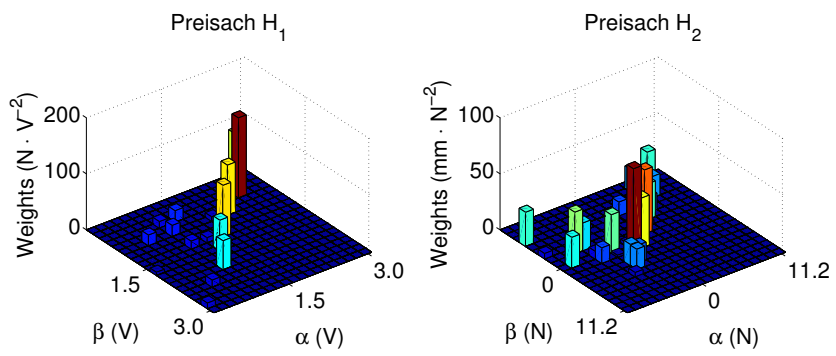

Fig. 9. Left figure: the identified weights of the Preisach term $H_{1}$. Right figure: the identified weights of the Preisach term $\mathrm{H}_{2}$ of the proposed model.

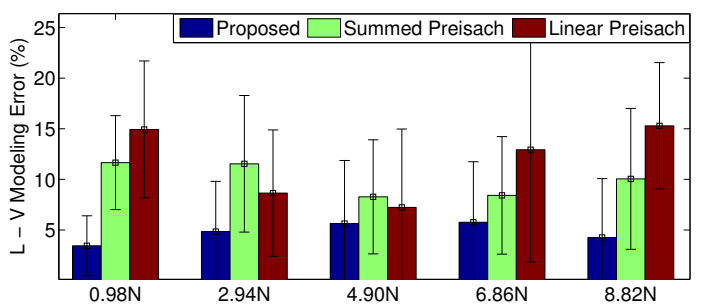

(a)

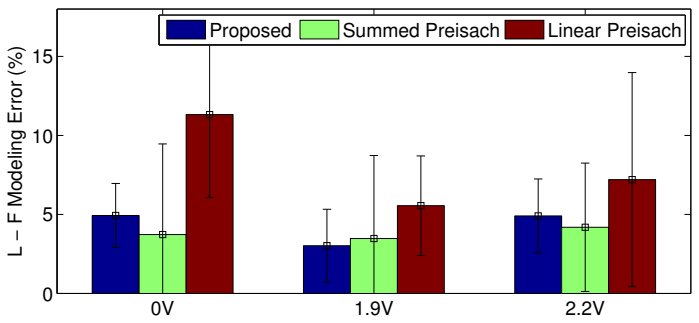

(b)

Fig. 10. (a). The Contraction Length - Temperature Surrogate hysteresis and (b) Contraction Length - Force hysteresis modeling error comparisons.

2) Summed Preisach: For a fair comparison, the discretization levels of the Preisach terms $\mathrm{H}_{3}$ and $\mathrm{H}_{4}$ are also chosen to be 20. Unlike the identification of the proposed model, $\mathrm{H}_{3}$ and $\mathrm{H}_{4}$ are directly identified based on Contraction Length - Temperature Surrogate hysteresis and Contraction Length Force hysteresis.

The modeling performance is shown in Fig. 7, Fig. 8 , and Fig. 10. The average and RMS errors for the Contraction Length - Temperature Surrogate hysteresis are $10.0 \%$ and $11.8 \%$, respectively. The average and RMS errors for the Contraction Length - Force hysteresis are $3.8 \%$ and $6.3 \%$, respectively. While this model demonstrates comparable mod- eling accuracy as the proposed model for Contraction Length - Force hysteresis, its modeling performance for Contraction Length - Temperature Surrogate hysteresis is much worse. This model cannot capture the hysteresis curve differences under different conditions, as shown in Fig. 7 and Fig. 8 Furthermore, due to the fact that the Force - Temperature Surrogate hysteresis is not directly employed for model identification; this model cannot easily describe the coupling between force and temperature surrogate.

3) Linear Preisach: The discretization level of $H_{5}$ is also chosen to be 20. The linear term is identified to be $a=3.34$ N/V. The modeling performance is shown in Fig. 6 (right), Fig. 7. Fig. 8, and Fig. 10 As discussed, albeit that the Force Temperature Surrogate hysteresis is less significant, the Linear Preisach model produces about $260 \%$ larger modeling error than that of the proposed model. The average and RMS errors for the Contraction Length - Temperature Surrogate hysteresis are $11.8 \%$ and $14.5 \%$, respectively. The average and RMS errors for the Contraction Length - Force hysteresis are $8.9 \%$ and $11.6 \%$, respectively. With the linear approximation, this model results in the largest error.

\section{Model Verification}

To further validate the effectiveness of the proposed model, separate experiments can be conducted to test each hysteresis relationship. Due to brevity purposes, this work only provide detailed verification results for the Contraction Length - Temperature Surrogate relationship. A randomly-chosen voltage input, as shown in Fig. 11.a), is applied to the SMA actuator under different loading forces. The experimental measurements of the steady-state contraction length values are shown in Fig. 11.b). Fig. 11.c) shows the contraction length estimation errors under each scheme. Since 18 different voltage steps are used in each loading condition, and 5 loading conditions are tested, a total of 90 data points are obtained. The corresponding average and RMS estimation errors are provided in Fig. 11. d). The effectiveness of the proposed model is further validated.

\section{CONClusion And Future Work}

This study proposes the first approach to capturing the threedimensional coupled hysteresis of robotic artificial muscles by embedding a two-stage Preisach model. Experimental results confirm that the proposed approach can effectively describe and estimate the hysteresis among temperature surrogate, contraction length, and force of SMA actuators.

This study is general in the following two aspects:

First, the proposed model can be constructed using any existing two-dimensional hysteresis models. The proposed scheme is not constrained to any hysteresis models. This has important practical usages since different embedding hysteresis elements can be chosen based on the application requirements and the properties of existing hysteresis models. For example, in cases where control accuracy is more important than computational and storage costs, the Preisach model with a large number of model parameters can be utilized; in 


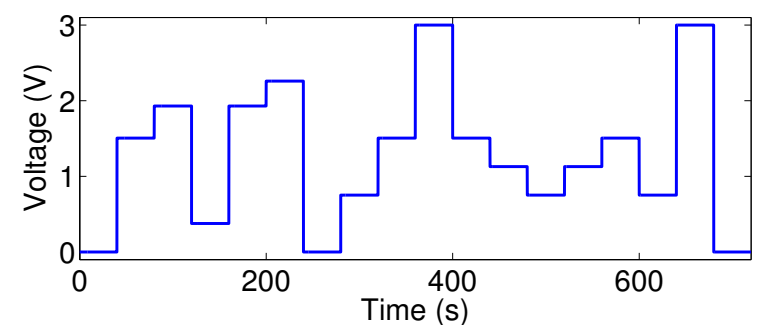

(a)

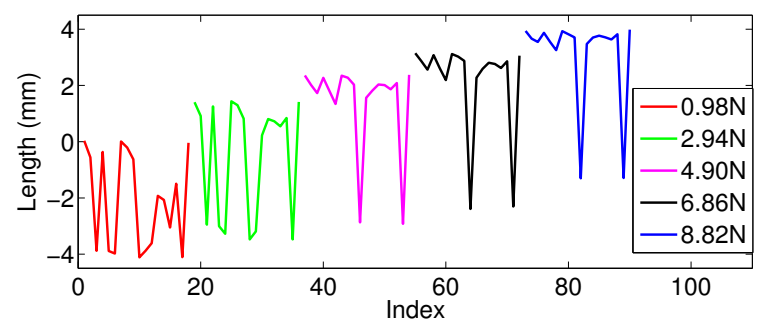

(b)

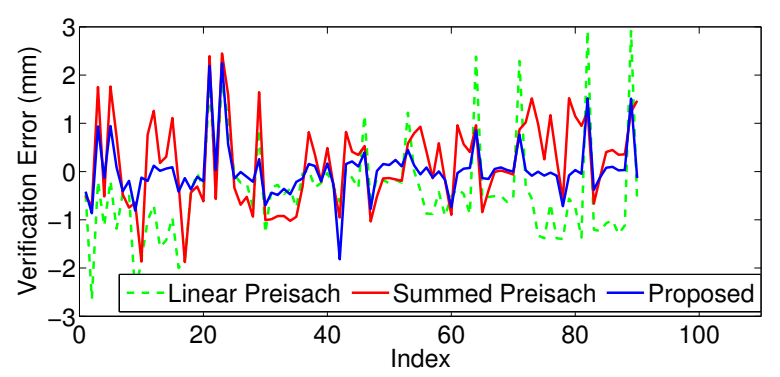

(c)

\begin{tabular}{llll}
\hline Methods & Proposed & Summed & Linear \\
\hline Average error & $4.7 \%$ & $9.3 \%$ & $10.4 \%$ \\
RMS error & $6.9 \%$ & $11.3 \%$ & $13.5 \%$ \\
\hline \multicolumn{4}{c}{ (d) }
\end{tabular}

Fig. 11. (a). A voltage input for model verification. (b). The corresponding experimental contraction length measurements. (c). Errors in contraction length predictions by the three modeling approaches. (d). The average error and RMS error comparisons of the three models.

cases where efficient implementation is more desirable, other hysteresis models that have simpler model structures can be employed, such as the Prandtl-Ishlinskii model and the BoucWen model.

Second, the proposed scheme is based on Preisach model, which is a phenomenological model that does not carry physical implications. Although for different behaviors, the identified model parameters will be different, the model formulation, identification, and implementation can all be similarly realized. This is the reason why the proposed study works for SMA actuators with other diameters and materials, as well as other types of robotic artificial muscles. However, physicsbased models are derived based on the physics of particular materials and structures, such model cannot be easily generalized. Like other phenomenological hysteresis models, the model parameters need to be identified for a particular system. By utilizing the relationship between the hysteresis profile and other actuator specifications (e.g., material, diameter, and length), the number of required experiments can be reduced.

Based on this study, future directions are envisioned in the following three areas:

First, the validation of the proposed model for other robotic artificial muscles is desirable. McKibben actuators can convert energy from compressed air to mechanical motion [3, 8]. Although they have been utilized in a variety of robotic applications [2, 5, 20], the accurate control of these actuators have proven to be difficult due to the friction-induced hysteresis [8]. Although static models have been derived, the complete hysteresis is often not explicitly captured [3, 11, 28]. SCP actuators can be created by continuously twisting carbon nanotube yarns, nylon fishing lines or sewing threads until coils were formed [7, 17]. This artificial muscle exhibits significant mechanical power, large contraction (20\%-50\% tensile actuations), and good dynamic range comparing to SMA actuators [7]. The existing methods either utilized complicated physical analysis [31] or simplified linear models [32]. The successful modeling and verification of the proposed model for these robotic artificial muscles and actual robotic systems can further strengthen this work.

Second, open-loop control of robotic artificial muscles can be realized. The proposed model can fully describe the quasistatic hysteresis of robotic artificial muscles in terms of input, strain, and force simultaneously. By inverting the proposed model, the inverted model can be adopted to compensate for the hysteresis in robotic artificial muscles and realize open-loop control. While the inverse of the Preisach model is available, there is no existing work on compensating the three-dimensional and coupled hysteresis in robotic artificial muscles. The complete inverse of the proposed model can be developed based on the two-stage embedding structure of the model.

Furthermore, by incorporating the dynamics of the robotic artificial muscles, closed-loop control of robotic artificial muscle-powered robots can be investigated. The hysteresis model considered in this paper is quasi-static and rateindependent, since it describes the steady-state behaviors of SMA actuators under a series of constant voltage steps. By incorporating the actuator dynamics and inverse compensation, the compensated system can be approximated as a linear dynamic system. Closed-loop control [30, 33] can be investigated by combining feedback with the inverse compensation schemes. In this way, real-time tracking control can be realized for artificial muscle-powered robots.

\section{ACKNOWLEDGMENTS}

We thank members of the Advanced Robotics and Controls Lab at University of California San Diego for intellectual discussions and technical help. We thank the Bioinspired Robotics and Design Lab directed by Michael T. Tolley at University of California San Diego for providing the image of the McKibben actuator (Fig. 1(b)). 


\section{REFERENCES}

[1] Mohammad Al Janaideh, Subhash Rakheja, and ChunYi Su. A generalized Prandtl-Ishlinskii model for characterizing the hysteresis and saturation nonlinearities of smart actuators Smart Materials and Structures, 18(4):045001, 2009. doi: 10.1088/0964-1726/18/ 4/045001. URL http://iopscience.iop.org/article/10.1088/ 0964-1726/18/4/045001/meta.

[2] A. T. Asbeck, S. M. M. De Rossi, I. Galiana, Y. Ding, and C. J. Walsh. Stronger, smarter, softer: Nextgeneration wearable robots IEEE Robotics \& Automation Magazine, 21(4):22-33, 2014. doi: 10.1109/MRA. 2014.2360283. URL http://ieeexplore.ieee.org/document/ 6990838/

[3] Ching-Ping Chou and B. Hannaford. Measurement and modeling of McKibben pneumatic artificial muscles IEEE Transactions on Robotics and Automation, 12(1):90-102, 1996. doi: 10.1109/70.481753. URL http://ieeexplore.ieee.org/document/481753/.

[4] Cheikh Cisse, Wael Zaki, and Tarak Ben Zineb. A review of constitutive models and modeling techniques for shape memory alloys International Journal of Plasticity, 76:244-284, 2016. doi: 10.1016/j.ijplas.2015.08. 006. URL http://www.sciencedirect.com/science/article/ pii/S0749641915001436.

[5] Raphael Deimel and Oliver Brock. A novel type of compliant, underactuated robotic hand for dexterous grasping. In Proceedings of Robotics: Science and Systems, Berkeley, USA, July 2014. doi: 10.15607/RSS.2014.X. 018. URL http://www.roboticsproceedings.org/rss10/p18. html.

[6] G. Finocchio, E. Cardelli, and B. Azzerboni. A simplified model for vector hysteresis computation IEEE Transactions on Magnetics, 42(4):955-958, 2006. doi: 10.1109/TMAG.2006.871991. URL http://ieeexplore. ieee.org/document/1608366/,

[7] Carter S. Haines, Márcio D. Lima, Na Li, Geoffrey M. Spinks, Javad Foroughi, John D. W. Madden, Shi Hyeong Kim, Shaoli Fang, Mônica Jung de Andrade, Fatma Göktepe, Özer Göktepe, Seyed M. Mirvakili, Sina Naficy, Xavier Lepró, Jiyoung Oh, Mikhail E. Kozlov, Seon Jeong Kim, Xiuru Xu, Benjamin J. Swedlove, Gordon G. Wallace, and Ray H. Baughman. Artificial muscles from fishing line and sewing thread Science, 343(6173):868-872, 2014. doi: 10.1126/science. 1246906. URL http://science.sciencemag.org/content/ 343/6173/868.

[8] R. V. Ham, T. G. Sugar, B. Vanderborght, K. W. Hollander, and D. Lefeber. Compliant actuator designs IEEE Robotics \& Automation Magazine, 16(3):81-94, 2009. doi: 10.1109/MRA.2009.933629. URL http://ieeexplore. ieee.org/document/5233419

[9] Mingyen Ho, Yeongjin Kim, Shing Shin Cheng, Rao Gullapalli, and Jaydev P. Desai. Design, development, and evaluation of an MRI-guided
SMA spring-actuated neurosurgical robot The International Journal of Robotics Research, 34(8): 1147-1163, 2015. doi: 10.1177/0278364915579069. URL https://www.ncbi.nlm.nih.gov/pmc/articles/ PMC4662428/pdf/nihms677690.pdf

[10] Jaronie Mohd Jani, Martin Leary, Aleksandar Subic, and Mark A. Gibson. A review of shape memory alloy research, applications and opportunities Materials \& Design, 56:1078-1113, 2014. doi: 10.1016/ j.matdes.2013.11.084. URL http://www.sciencedirect. com/science/article/pii/S0261306913011345.

[11] B. S. Kang, C. S. Kothera, B. K. S. Woods, and N. M. Wereley. Dynamic modeling of McKibben pneumatic artificial muscles for antagonistic actuation. In Proceedings of IEEE International Conference on Robotics and Automation (ICRA), pages 182-187, 2009. doi: 10.1109/ROBOT.2009.5152280. URL http://ieeexplore. ieee.org/document/5152280/

[12] Atilla Kilicarslan, Gangbing Song, and Karolos Grigoriadis. Modeling and hysteresis compensation in a thin SMA wire using ANFIS methods. Journal of Intelligent Material Systems and Structures, 22(1):45-57, 2011. doi: 10.1177/1045389X10392610. URL http://journals. sagepub.com/doi/abs/10.1177/1045389X10392610

[13] Pavel Krejci and Jurgen Sprekels. On a class of multi-dimensional Prandtl-Ishlinskii operators Physica B: Condensed Matter, 306(14):185-190, 2001. doi: 10.1016/S0921-4526(01)01001-8. URL http://www.sciencedirect.com/science/article/pii/ S0921452601010018

[14] Cecilia Laschi, Barbara Mazzolai, and Matteo Cianchetti. Soft robotics: Technologies and systems pushing the boundaries of robot abilities Science Robotics, 1(1), 2016. doi: 10.1126/scirobotics.aah3690. URL http: //robotics.sciencemag.org/content/1/1/eaah3690

[15] K. K. Leang, Q. Zou, and S. Devasia. Feedforward control of piezoactuators in atomic force microscope systems IEEE Control Systems, 29(1):70-82, 2009. doi: 10.1109/MCS.2008.930922. URL http://ieeexplore.ieee. org/document/4753744/

[16] D.J. Leo. Engineering analysis of smart material systems Wiley InterScience. Wiley, 2007. ISBN 9780471684770. URL http://www.wiley.com/ WileyCDA/WileyTitle/productCd-0471684775.html

[17] Márcio D. Lima, Na Li, Mônica Jung de Andrade, Shaoli Fang, Jiyoung Oh, Geoffrey M. Spinks, Mikhail E. Kozlov, Carter S. Haines, Dongseok Suh, Javad Foroughi, Seon Jeong Kim, Yongsheng Chen, Taylor Ware, Min Kyoon Shin, Leonardo D. Machado, Alexandre F. Fonseca, John D. W. Madden, Walter E. Voit, Douglas S. Galvão, and Ray H. Baughman. Electrically, chemically, and photonically powered torsional and tensile actuation of hybrid carbon nanotube yarn muscles Science, 338(6109):928-932, 2012. doi: 10.1126/science. 1226762. URL http://science.sciencemag.org/content/ $338 / 6109 / 928$ 
[18] I. MacKenzie and D. L. Trumper. Real-time hysteresis modeling of a reluctance actuator using a shearedhysteresis-model observer. IEEE/ASME Transactions on Mechatronics, 21(1):4-16, 2016. doi: 10.1109/TMECH. 2015.2513769. URL http://ieeexplore.ieee.org/document/ 7372456/.

[19] I.D. Mayergoyz. Mathematical models of hysteresis and their applications. Springer-Verlag, 1991. URL http: //www.sciencedirect.com/science/book/9780124808737

[20] Yong-Lae Park, Bor rong Chen, Nestor O PerezArancibia, Diana Young, Leia Stirling, Robert J Wood, Eugene C Goldfield, and Radhika Nagpal. Design and control of a bio-inspired soft wearable robotic device for ankle-foot rehabilitation. Bioinspiration \& Biomimetics, 9(1):016007, 2014. doi: 10.1088/1748-3182/9/1/016007. URL http://iopscience.iop.org/1748-3190/9/1/016007

[21] M. Ruderman and T. Bertram. Control of Magnetic Shape Memory Actuators Using Observer-Based Inverse Hysteresis Approach IEEE Transactions on Control Systems Technology, 22(3):1181-1189, 2014. doi: 10. 1109/TCST.2013.2271354. URL http://ieeexplore.ieee. org/document/6558803/.

[22] Daniela Rus and Michael T. Tolley. Design, fabrication and control of soft robots Nature, 521, 2015. doi: 10. 1038/nature14543. URL http://www.nature.com/nature/ journal/v521/n7553/abs/nature14543.html.

[23] S. Seok, C. D. Onal, K. J. Cho, R. J. Wood, D. Rus, and S. Kim. Meshworm: A peristaltic soft robot with antagonistic nickel titanium coil actuators IEEE/ASME Transactions on Mechatronics, 18(5):1485-1497, 2013. doi: 10.1109/TMECH.2012.2204070. URL http://ieeexplore. ieee.org/document/6232458/.

[24] Zhiyong Sun, Bo Song, Ning Xi, Ruiguo Yang, Lina Hao, and Liangliang Chen. Compensating asymmetric hysteresis for nanorobot motion control In Proceedings of IEEE International Conference on Robotics and Automation (ICRA), pages 3501-3506, 2015. doi: 10.1109/ICRA. 2015.7139683. URL http://ieeexplore.ieee.org/document/ 7139683/

[25] X Tan and J Baras. Modeling and control of hysteresis in magnetostrictive actuators Automatica, 40(9): 1469-1480, 2004 . doi: 10.1016/j.automatica.2004.04. 006. URL http://www.sciencedirect.com/science/article/ pii/S0005109804001244

[26] Bertrand Tondu. What is an artificial muscle? A systemic approach Actuators, 4(4):336, 2015. doi: 10.3390/ act4040336. URL http://www.mdpi.com/2076-0825/4/4/ 336.

[27] B. Vanderborght, A. Albu-Schaeffer, A. Bicchi, E. Burdet, D.G. Caldwell, R. Carloni, M. Catalano, O. Eiberger, W. Friedl, G. Ganesh, M. Garabini, M. Grebenstein, G. Grioli, S. Haddadin, H. Hoppner, A. Jafari, M. Laffranchi, D. Lefeber, F. Petit, S. Stramigioli, N. Tsagarakis, M. Van Damme, R. Van Ham, L.C. Visser, and S. Wolf. Variable impedance actuators: A review Robotics and Autonomous Systems,
61(12):1601-1614, 2013. doi: 10.1016/j.robot.2013.06. 009. URL http://www.sciencedirect.com/science/article/ pii/S0921889013001188.

[28] T. Vo-Minh, T. Tjahjowidodo, H. Ramon, and H. Van Brussel. A new approach to modeling hysteresis in a pneumatic artificial muscle using The Maxwell-Slip model IEEE/ASME Transactions on Mechatronics, 16 (1):177-186, 2011. doi: 10.1109/TMECH.2009.2038373. URL http://ieeexplore.ieee.org/document/5382520/.

[29] T. Wang, Z. Shi, D. Liu, C. Ma, and Z. Zhang. An accurately controlled antagonistic shape memory alloy actuator with self-sensing. Sensors, 12(6):76827700, 2012. doi: 10.3390/s120607682. URL http://www.mdpi. com/1424-8220/12/6/7682.

[30] Q. Xu. Adaptive discrete-time sliding mode impedance control of a piezoelectric microgripper. IEEE Transactions on Robotics, 29(3):663-673, 2013. doi: 10. 1109/TRO.2013.2239554. URL http://ieeexplore.ieee. org/document/6423296/

[31] Qianxi Yang and Guoqiang Li. A top-down multi-scale modeling for actuation response of polymeric artificial muscles Journal of the Mechanics and Physics of Solids, 92:237-259, 2016. doi: 10.1016/j.jmps.2016.04. 007. URL http://www.sciencedirect.com/science/article/ pii/S0022509615303537.

[32] Michael Yip and G. Niemeyer. High-performance robotic muscles from conductive nylon sewing thread In Proceedings 2015 IEEE International Conference on Robotics and Automation (ICRA), pages 2313-2318, 2015. doi: 10.1109/ICRA.2015.7139506. URL http: //ieeexplore.ieee.org/document/7139506/.

[33] Mohammad Reza Zakerzadeh and Hassan Sayyaadi. Precise position control of shape memory alloy actuator using inverse hysteresis model and model reference adaptive control system Mechatronics, 23(8): 1150-1162, 2013. doi: 10.1016/j.mechatronics.2013.10. 001. URL http://www.sciencedirect.com/science/article/ pii/S0957415813001694.

[34] J. Zhang, E. Merced, N. Sepúlveda, and X. Tan. Modeling and inverse compensation of non-monotonic hysteresis in $\mathrm{VO}_{2}$-coated microactuators IEEE/ASME Transactions on Mechatronics, 19(2):579-588, 2014. doi: 10.1109/TMECH.2013.2250989. URL http://ieeexplore. ieee.org/document/6482641/

[35] J Zhang, D Torres, J Ebel, N Sepúlveda, and X Tan. A composite hysteresis model in self-sensing feedback control of fully integrated $\mathrm{VO}_{2}$ microactuators IEEE/ASME Transactions on Mechatronics, 21(5):24052417, 2016. doi: 10.1109/TMECH.2016.2569499. URL http://ieeexplore.ieee.org/document/7470470/.

[36] Jun Zhang, Kaushik Iyer, Anthony Simeonov, and Michael C. Yip. Modeling and inverse compensation of hysteresis in super-coiled polymer artificial muscles IEEE Robotics and Automation Letters, 2(2):773-780, 2017. doi: 10.1109/LRA.2017.2651401. URL http: //www.ieeeexplore.ws/document/7814286/ 